\title{
Enhancement of Gravitational Waves Induced by Scalar Perturbations due to a Sudden Transition from an Early Matter Era to the Radiation Era
}

\author{
Keisuke Inomata, ${ }^{1,2}$ Kazunori Kohri, ${ }^{2,3,4}$ Tomohiro Nakama, ${ }^{5}$ and Takahiro Terada ${ }^{3}$ \\ ${ }^{1}$ ICRR, University of Tokyo, Kashiwa, 277-8582, Japan \\ ${ }^{2}$ Kavli IPMU (WPI), UTIAS, University of Tokyo, Kashiwa, 277-8583, Japan \\ ${ }^{3}$ Theory Center, IPNS, KEK, 1-1 Oho, Tsukuba, Ibaraki 305-0801, Japan \\ ${ }^{4}$ The Graduate University for Advanced Studies (SOKENDAI), 1-1 Oho, Tsukuba, Ibaraki 305-0801, Japan \\ ${ }^{5}$ Institute for Advanced Study, The Hong Kong University of Science and Technology, \\ Clear Water Bay, Kowloon, Hong Kong
}

(Dated: October 7, 2022)

\begin{abstract}
We study gravitational waves induced from the primordial scalar perturbations at second order around the reheating of the Universe. We consider reheating scenarios in which a transition from an early matter dominated era to the radiation dominated era completes within a timescale much shorter than the Hubble time at that time. We find that an enhanced production of induced gravitational waves occurs just after the reheating transition because of fast oscillations of scalar modes well inside the Hubble horizon. This enhancement mechanism just after an early matterdominated era is much more efficient than a previously known enhancement mechanism during an early matter era, and we show that the induced gravitational waves could be detectable by future observations if the reheating temperature $T_{\mathrm{R}}$ is in the range $T_{\mathrm{R}} \lesssim 7 \times 10^{-2} \mathrm{GeV}$ or $20 \mathrm{GeV} \lesssim T_{\mathrm{R}} \lesssim$ $2 \times 10^{7} \mathrm{GeV}$. This is the case even if the scalar perturbations on small scales are not enhanced relative to those on large scales, probed by the observations of the cosmic microwave background.
\end{abstract}

\section{INTRODUCTION}

Recently, gravitational waves (GWs) have been attracting more and more attention. So far, LIGO and Virgo collaborations have succeeded in detecting GWs from merging black holes [1,2]. KAGRA is also expected to detect GWs in a few years [3]. GWs provide a lot of information about not only the nature and the origins of black holes, but also about the early Universe. Stochastic GWs induced by curvature perturbations at second order are one of the cosmological GW sources closely related to the study of the early Universe [4-8]. There are a number of recent studies about such induced GWs [922], some of which are related to primordial black holes. The induced GWs, along with other GW backgrounds of astrophysical as well as cosmological origins, can be investigated by the ongoing or future GW projects, such as pulsar timing array observations (EPTA [23], PPTA [24], NANOGrav [25], SKA [26, 27]), ground based interferometer experiments (advanced LIGO (aLIGO) [28], Virgo [29], KAGRA [3], Einstein Telescope (ET) [26, 30, 31], Cosmic Explorer [32]), and space based interferometer experiments (LISA [26, 30, 33], DECIGO [34, 35], BBO [35, 36]). Future measurements of stochastic GWs can be a key to reveal the evolution history of the Universe.

In this work, we focus on the relation between the induced GWs and an early matter-dominated era (eMD era). An eMD era is a period during which the energy density of a massive field dominates the Universe before the reheating. Although the eMD effects on the induced GWs have been discussed in Refs. [10, 37, 38], in our accompanying paper [39], we find that if we carefully take into account the evolution of the gravitational potential, which is the source of the induced GWs, around the transition from an eMD era to the radiation dominated era (RD era), the predictions for the induced GWs can change. In particular, we show that the induced GWs can be significantly suppressed for a gradual transition, whose transition timescale is comparable to the Hubble time at that time. In some cosmological scenarios (see Appendix A) however, the transition from an eMD era to the $\mathrm{RD}$ era is sudden, i.e. the timescale of the transition is much shorter than the Hubble time at that time. The purpose of this paper is to study the induced GWs in such sudden transition cases.

The effects on the induced GWs of a sudden reheating mainly arise during the RD era by the scalar perturbations that have already entered the horizon during an eMD era. Although GWs induced during an eMD era have been studied in Refs. [10, 37, 38], GWs induced during the $\mathrm{RD}$ era by the perturbations experiencing an eMD era on subhorizon scales have not been investigated in the previous studies. However, we point out that, in sudden-reheating scenarios, GWs induced during the RD era can be larger than GWs induced during an eMD era by several orders of magnitude. Making use of this enhancement, we might be able to determine the reheating temperature by future GW detectors, as we discuss later.

We begin by reviewing the equations to calculate the induced GWs in Sec. II, and based on these equations we obtain the power spectra for the induced GWs in Sec. III assuming a sudden transition from an eMD era to the RD era. We explore possibilities to determine the reheating temperature in Sec. IV by making use of the enhancement of the induced GWs associated with a sudden reheating, and Sec. V is dedicated to conclusions. In Appendix A, we discuss two models that can realize a sudden transition from an eMD era to the RD era, and in Appendix $\mathrm{B}$ we present approximate formulas for the induced GWs 
in sudden-reheating scenarios.

\section{FORMULAS FOR INDUCED GRAVITATIONAL WAVES}

In the following, we briefly review the equations to calculate induced GWs (see Ref. [10] for more details). We assume that an eMD era ended abruptly with the Universe entering into the RD era at a conformal time $\eta=\eta_{\mathrm{R}}$. Then the scale factor and the conformal Hubble parameter are given by

$$
\frac{a(\eta)}{a\left(\eta_{\mathrm{R}}\right)}=\left\{\begin{array}{ll}
\left(\frac{\eta}{\eta_{\mathrm{R}}}\right)^{2} \\
2 \frac{\eta}{\eta_{\mathrm{R}}}-1
\end{array} \quad, \quad \mathcal{H}(\eta)=\left\{\begin{array}{ll}
\frac{2}{\eta} & \left(\eta \leq \eta_{\mathrm{R}}\right) \\
\frac{1}{\eta-\eta_{\mathrm{R}} / 2} & \left(\eta>\eta_{\mathrm{R}}\right)
\end{array} .\right.\right.
$$

We also assume that the curvature perturbations follow a Gaussian distribution ${ }^{1}$ and adopt the conformal Newtonian gauge ${ }^{2}$ for simplicity. Since we focus on the effects of an eMD era, relevant to very small-scale fluctuations $\left(k \gg k_{\text {eq }}=0.0103 \mathrm{Mpc}^{-1}[44]\right)$, in this paper we do not consider the enhancement of the induced GWs during the late MD era $(z \lesssim 3400)[5,45]$.

The energy density parameter of GWs per logarithmic interval in $k$ is given by

$$
\begin{aligned}
\Omega_{\mathrm{GW}}(\eta, k) & =\frac{\rho_{\mathrm{GW}}(\eta, k)}{\rho_{\mathrm{tot}}(\eta)} \\
& =\frac{1}{24}\left(\frac{k}{a(\eta) H(\eta)}\right)^{2} \overline{\mathcal{P}_{h}(\eta, k)}
\end{aligned}
$$

where $\overline{\mathcal{P}_{h}(\eta, k)}$ is the time averaged power spectrum of GWs. It can be evaluated from the power spectrum $\mathcal{P}_{\zeta}$ of the curvature perturbations by [10]

$$
\begin{aligned}
\overline{\mathcal{P}_{h}(\eta, k)}=4 \int_{0}^{\infty} \mathrm{d} v & \int_{|1-v|}^{1+v} \mathrm{~d} u\left(\frac{4 v^{2}-\left(1+v^{2}-u^{2}\right)^{2}}{4 v u}\right)^{2} \\
& \times \overline{I^{2}\left(u, v, k, \eta, \eta_{\mathrm{R}}\right)} \mathcal{P}_{\zeta}(u k) \mathcal{P}_{\zeta}(v k) .
\end{aligned}
$$

Here, $I\left(u, v, k, \eta, \eta_{\mathrm{R}}\right)$, describing the time dependence of GWs, is given by

$$
I\left(u, v, k, \eta, \eta_{\mathrm{R}}\right)=\int_{0}^{x} \mathrm{~d} \bar{x} \frac{a(\bar{\eta})}{a(\eta)} k G_{k}(\eta, \bar{\eta}) f\left(u, v, \bar{x}, x_{\mathrm{R}}\right),
$$

where $x$ and $x_{\mathrm{R}}$ are defined as $x \equiv k \eta$ and $x_{\mathrm{R}} \equiv k \eta_{\mathrm{R}}$. In this equation, $G_{k}$ is the Green's function being the solution of

$$
G_{k}^{\prime \prime}(\eta, \bar{\eta})+\left(k^{2}-\frac{a^{\prime \prime}(\eta)}{a(\eta)}\right) G_{k}(\eta, \bar{\eta})=\delta(\eta-\bar{\eta})
$$

where a prime denotes differentiation with respect to $\eta$, not $\bar{\eta}$. Note that the concrete expression of $G_{k}$ depends on the background evolution of the Universe, which is an eMD era or the RD era in our problem. In addition, $f\left(u, v, \bar{x}, x_{\mathrm{R}}\right)$ is the source function defined as

$$
f\left(u, v, \bar{x}, x_{\mathrm{R}}\right)=\frac{3\left(2(5+3 w) \Phi(u \bar{x}) \Phi(v \bar{x})+4 \mathcal{H}^{-1}\left(\Phi^{\prime}(u \bar{x}) \Phi(v \bar{x})+\Phi(u \bar{x}) \Phi^{\prime}(v \bar{x})\right)+4 \mathcal{H}^{-2} \Phi^{\prime}(u \bar{x}) \Phi^{\prime}(v \bar{x})\right)}{25(1+w)}
$$

where $\omega=P / \rho$ is the equation-of-state parameter with $P$ and $\rho$ being the pressure and the energy density, respectively. $\Phi$ is the transfer function of the gravitational potential, which satisfies $\Phi\left(x \rightarrow 0, x_{\mathrm{R}}\right) \rightarrow 1$, and a prime denotes differentiation with respect to the conformal time, that is, $\Phi^{\prime}(u \bar{x}) \equiv \partial \Phi(u \bar{x}) / \partial \bar{\eta}=u k \partial \Phi(u \bar{x}) / \partial(u \bar{x})$. The second argument of $\Phi$ is abbreviated in Eq. (6) for compact notation. $\Phi(u \bar{x})$ actually means $\Phi\left(u \bar{x}, u \bar{x}_{\mathrm{R}}\right)$, and $\Phi(v \bar{x})$ should be understood similarly.

The evolution equation for $\Phi$ is [46]

$$
\Phi^{\prime \prime}+3(1+w) \mathcal{H} \Phi^{\prime}+w k^{2} \Phi=0 .
$$

\footnotetext{
${ }^{1} \mathrm{GWs}$ induced by the curvature perturbations with nonGaussianity are discussed in Refs. [11, 14, 40, 41]

2 The gauge dependence of induced GWs is discussed in Refs. [42, 43].
}

By solving this equation, we find

$$
\Phi\left(x, x_{\mathrm{R}}\right)= \begin{cases}1 & \left(\text { for } x \leq x_{\mathrm{R}}\right), \\ A\left(x_{\mathrm{R}}\right) \mathcal{J}(x)+B\left(x_{\mathrm{R}}\right) \mathcal{Y}(x) & \left(\text { for } x \geq x_{\mathrm{R}}\right),\end{cases}
$$

where we have dropped the decaying mode for $\eta<\eta_{\mathrm{R}}$. In this expression, $\mathcal{J}(x)$ and $\mathcal{Y}(x)$ are defined from the first and second spherical Bessel functions, $j_{1}(x)$ and $y_{1}(x)$, as

$$
\begin{aligned}
& \mathcal{J}(x)=\frac{3 \sqrt{3} j_{1}\left(\frac{x-x_{\mathrm{R}} / 2}{\sqrt{3}}\right)}{x-x_{\mathrm{R}} / 2}, \\
& \mathcal{Y}(x)=\frac{3 \sqrt{3} y_{1}\left(\frac{x-x_{\mathrm{R}} / 2}{\sqrt{3}}\right)}{x-x_{\mathrm{R}} / 2},
\end{aligned}
$$

and the coefficients $A\left(x_{\mathrm{R}}\right)$ and $B\left(x_{\mathrm{R}}\right)$ are determined so 
that $\Phi(x)$ and $\Phi^{\prime}(x)$ are continuous at $x=x_{\mathrm{R}}$ :

$$
\begin{aligned}
& A\left(x_{\mathrm{R}}\right)=\frac{1}{\mathcal{J}\left(x_{\mathrm{R}}\right)-\frac{\mathcal{Y}\left(x_{\mathrm{R}}\right)}{\mathcal{Y}^{\prime}\left(x_{\mathrm{R}}\right)} \mathcal{J}^{\prime}\left(x_{\mathrm{R}}\right)}, \\
& B\left(x_{\mathrm{R}}\right)=-\frac{\mathcal{J}^{\prime}\left(x_{\mathrm{R}}\right)}{\mathcal{Y}^{\prime}\left(x_{\mathrm{R}}\right)} A\left(x_{\mathrm{R}}\right) .
\end{aligned}
$$

In Appendix A, we introduce a model realizing a suddenreheating transition and check that the above analytic expression for $\Phi$ with these connection conditions coincides well with the numerical solution for $\Phi$ calculated for that model.

We can reexpress Eq. (4) as in Ref. [10] with a slight refinement of the time dependence of the scale factor:

$$
\begin{aligned}
I\left(u, v, x, x_{\mathrm{R}}\right)=\int_{0}^{x_{\mathrm{R}}} \mathrm{d} \bar{x} & \left(\frac{1}{2\left(x / x_{\mathrm{R}}\right)-1}\right)\left(\frac{\bar{x}}{x_{\mathrm{R}}}\right)^{2} \\
& \times k G_{k}^{\mathrm{eMD} \rightarrow \mathrm{RD}}(\eta, \bar{\eta}) f\left(u, v, \bar{x}, x_{\mathrm{R}}\right) \\
+ & \int_{x_{\mathrm{R}}}^{x} \mathrm{~d} \bar{x}\left(\frac{2\left(\bar{x} / x_{\mathrm{R}}\right)-1}{2\left(x / x_{\mathrm{R}}\right)-1}\right) \\
\quad & \times k G_{k}^{\mathrm{RD}}(\eta, \bar{\eta}) f\left(u, v, \bar{x}, x_{\mathrm{R}}\right) \\
\equiv & I_{\mathrm{eMD}}\left(u, v, x, x_{\mathrm{R}}\right)+I_{\mathrm{RD}}\left(u, v, x, x_{\mathrm{R}}\right),
\end{aligned}
$$

where $I_{\mathrm{eMD}}$ and $I_{\mathrm{RD}}$ represent the contributions from GWs induced during an eMD era and the RD era, respectively. See our accompanying paper [39] for the concrete expressions of the Green functions. We approximate $\overline{I^{2}\left(u, v, x, x_{\mathrm{R}}\right)}$ in Eq. (3) as

$$
\overline{I^{2}\left(u, v, x, x_{\mathrm{R}}\right)} \simeq \overline{I_{\mathrm{eMD}}^{2}\left(u, v, x, x_{\mathrm{R}}\right)}+\overline{I_{\mathrm{RD}}^{2}\left(u, v, x, x_{\mathrm{R}}\right)} .
$$

Correspondingly, we approximately split $\Omega_{\mathrm{GW}}$ into two parts as $\Omega_{\mathrm{GW}} \simeq \Omega_{\mathrm{GW}, \mathrm{RD}}+\Omega_{\mathrm{GW}, \mathrm{eMD}}$, where $\Omega_{\mathrm{GW}, \mathrm{RD}}$ and $\Omega_{\mathrm{GW}, \mathrm{eMD}}$ are calculated from $\overline{I_{\mathrm{RD}}^{2}}$ and $\overline{I_{\mathrm{eMD}}^{2}}$, respectively.

The analytic formulas for $I_{\mathrm{eMD}}$ and $I_{\mathrm{RD}}$ are derived in Ref. [10]. In this reference, we adopted an implicit assumption that GWs induced during the RD era by the perturbations having entered the horizon during an eMD era, which we focus on in this work, are subdominant compared to the GWs 1) which are induced during the eMD era and 2) which are induced by the perturbations entering the horizon after the reheating. However, this assumption is not true in realistic situations. In our accompanying paper [39], we consider a gradual reheating transition and show that the contributions from $I_{\mathrm{RD}}$ play an important roll for the suppression of induced GWs. In addition, in a sudden-reheating scenario, the dominant contribution comes from $I_{\mathrm{RD}}$ as we will show in Sec. III.

During the RD era, the gravitational potential, the source of GWs, decays on subhorizon scales, and therefore $\Omega_{\mathrm{GW}}$ becomes constant after the gravitational potential has sufficiently decayed. Here, we define $\eta_{c}$ as the moment when $\Omega_{\mathrm{GW}}$ becomes constant. Note that since we focus on small scales, where the effects of an eMD era may leave traces, $\eta_{c}$ is well before the standard matterradiation equality time. Taking into account the evolution of GWs during the late MD era and the change in the effective relativistic degrees of freedom, we can write the current energy density parameter $\Omega_{\mathrm{GW}}\left(\eta_{0}, k\right)$ as [47]

$$
\Omega_{\mathrm{GW}}\left(\eta_{0}, k\right)=0.39\left(\frac{g_{c}}{106.75}\right)^{-1 / 3} \Omega_{\mathrm{r}, 0} \Omega_{\mathrm{GW}}\left(\eta_{c}, k\right),
$$

where $\Omega_{\mathrm{r}, 0}$ is the current value of the energy density parameter for radiation. In this paper, we denote the effective relativistic degrees of freedom by $g$, and $g_{c}$ in this equation is its value at $\eta=\eta_{c}$.

\section{CALCULATIONS OF INDUCED GRAVITATIONAL WAVES}

Using the above equations and the analytic formulas in Ref. [10], we calculate induced GWs. To be specific, we assume the following power spectrum of the curvature perturbation:

$$
\mathcal{P}_{\zeta}(k)=\Theta\left(k_{\max }-k\right) A_{s}\left(\frac{k}{k_{*}}\right)^{n_{s}-1},
$$

where $A_{s}$ is the amplitude at the pivot scale $k_{*}, n_{s}$ is the tilt of the power spectrum, and $\Theta$ is the Heaviside step function. We introduce $k_{\max }$ as the cutoff scale of the power spectrum. Since matter density perturbations grow in proportion to the scale factor during a MD era, perturbations may enter into the non-linear regime, depending on the amplitude of primordial fluctuations and the duration of an eMD era. If perturbations remain in the linear regime during an eMD era, the cutoff scale is the Hubble radius at the beginning of an eMD era. On the other hand if such nonlinearities arise, $k_{\max }$ should be chosen as the wavenumber of perturbations that are entering the non-linear regime at the end of an eMD era, since our formalism is based on the linear theory. ${ }^{3}$ In this case, the smallest scale on which we can apply the linear theory is approximated as [39]

$$
k_{\mathrm{NL}} \sim 470 / \eta_{\mathrm{R}} .
$$

Hence, to ensure the validity of our analysis, we limit our analysis to cases with $k_{\max } \leq 450 / \eta_{\mathrm{R}}$. That is, we do not take into account GWs that are generated from nonlinear perturbations, and this means our analysis would lead to conservative estimations of the GW spectrum.

Figure 1 shows the scale dependence of the spectrum of induced GWs. In this figure, we take $n_{s}=1$ for simplicity. We can see that $\Omega_{\mathrm{GW}, \mathrm{RD}}$ is much larger than $\Omega_{\mathrm{GW}, \mathrm{eMD}}$. This is because the GWs induced during the

\footnotetext{
3 There are works discussing GWs induced by non-linear perturbations, though the results inevitably involve some uncertainties $[48,49]$.
} 
RD era by the subhorizon perturbations that entered the horizon during an eMD era, neglected in the previous works but taken into account in this work, are significant. For comparison, we also plot the GW spectra induced by the power spectra of $\mathcal{P}(k)=\Theta\left(k_{\max }-k\right) \Theta\left(k-0.7 k_{\max }\right) A_{s}$ and $\mathcal{P}(k)=\Theta\left(0.7 k_{\max }-k\right) \Theta\left(k-0.4 k_{\max }\right) A_{s}$ with blue and red lines. As shown in the figure, the contributions from the smallest scales (blue dashed line) are the dominant contributions to the total spectrum (solid black line) except for the large-scale-side tail of the sharp peak $\left(0.3 \lesssim k / k_{\max } \lesssim 1\right)$. This sharp peak is due to the resonance effect, which is a characteristic feature of GWs induced during the $\mathrm{RD}$ era [4], when the gravitational potential oscillates. The tail of the sharp peak is formed by the envelope of the resonance effects on these scales (see the red dot-dashed line). In this way, the spectrum of the induced GWs is produced dominantly by the smallest scales, and the resonant amplification plays a key role. This understanding becomes clearer in Appendix B, where we derive approximate analytic formulas for induced GWs for sudden-reheating scenarios. On much larger scales, the contributions from the perturbations entering the horizon after the reheating dominate induced GWs, whose spectrum becomes scale invariant $\Omega_{\mathrm{GW}}\left(\eta_{c}, k\right) \simeq 0.8222 A_{s}^{2}[10]$. This can be observed in the GW spectrum for $k<10^{9} \mathrm{Mpc}^{-1}$ in Fig. 2, though in that figure a slightly scale-dependent primordial spectrum is assumed, leading to a slight scale dependence of $\Omega_{\mathrm{GW}}$.

The main reason why induced GWs are enhanced is that the gravitational potential $\Phi$ with large $k\left(\gg 1 / \eta_{\mathrm{R}}\right)$ is constant until $\eta=\eta_{\mathrm{R}}$ and, after the reheating, it oscillates with the timescale $\sim 1 / k$, much shorter than its decay timescale $\sim \eta_{\mathrm{R}}$. Due to the fast oscillations of perturbations with unsuppressed amplitudes, which remained constant until the moment of the reheating, induced GWs are significantly enhanced. ${ }^{4}$ Note that the dominant contributions come from the last term in Eq. (6), which involves two time derivatives of the gravitational potential. This is because, at the beginning of the RD era, the last term can be approximated as $\mathcal{H}^{-2} \Phi^{\prime} \Phi^{\prime} \sim\left(k \eta_{\mathrm{R}}\right)^{2} \Phi^{2} \gg \Phi^{2}$ for the perturbations that entered the horizon well before the sudden reheating. In other words, the factor $\left(k \eta_{\mathrm{R}}\right)^{2}$ in the source term and the amplitude of $\Phi$ that remained constant until the reheating are the main causes for the enhancement.

In addition to numerical solutions, we also obtain approximate analytic formulas of $\Omega_{\mathrm{GW}, \mathrm{RD}}$ in Appendix B, with $\Omega_{\mathrm{GW}, \mathrm{RD}}$ given by the sum of Eqs. (B5) and (B7).

\footnotetext{
4 Although the perturbations entering the horizon during the RD era also oscillate with the timescale much shorter than their decay timescale well after (not soon after) the horizon entry, the amplitudes of the perturbations start to decay soon after the horizon entry, unlike during an eMD era, and therefore the enhancement is not caused by the perturbations entering the horizon during the RD era.
}

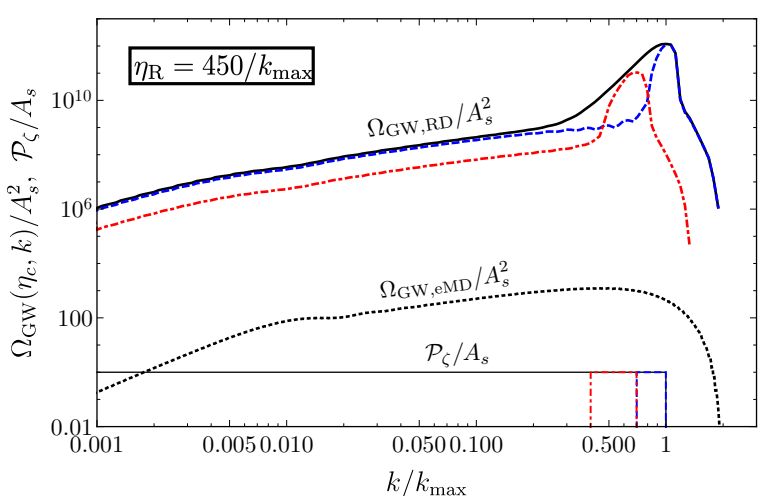

FIG. 1. Energy density parameters of GWs, for each logarithmic interval of wavenumber, induced during the RD era $\left(\Omega_{\mathrm{GW}, \mathrm{RD}}\left(\eta_{c}, k\right)\right)$ and during an eMD era $\left(\Omega_{\mathrm{GW}, \mathrm{eMD}}\left(\eta_{c}, k\right)\right)$, as well as the power spectrum $\mathcal{P}_{\zeta}(k)$ of curvature perturbations. They are normalized by $A_{s}^{2}$ or $A_{s}$, respectively, and we take $\eta_{\mathrm{R}}=450 / k_{\max }$. The black lines are derived from $\mathcal{P}_{\zeta}(k)=$ $\Theta\left(k_{\max }-k\right) A_{s}$. For comparison, the blue and red lines are also shown, which are derived from $\mathcal{P}_{\zeta}(k)=\Theta\left(k_{\max }-k\right) \Theta(k-$ $\left.0.7 k_{\max }\right) A_{s}$ and $\mathcal{P}_{\zeta}(k)=\Theta\left(0.7 k_{\max }-k\right) \Theta\left(k-0.4 k_{\max }\right) A_{s}$, respectively.

Using these expressions, the GW spectrum is roughly expressed as

$$
\frac{\Omega_{\mathrm{GW}}\left(\eta_{c}, k\right)}{A_{\mathrm{s}}^{2}} \simeq\left\{\begin{array}{ll}
0.8 & \left(x_{\mathrm{R}} \lesssim 150 x_{\max , \mathrm{R}}^{-5 / 3}\right) \\
3 \times 10^{-7} x_{\mathrm{R}}^{3} x_{\max , \mathrm{R}}^{5} & \left(150 x_{\max , \mathrm{R}}^{-5 / 3} x_{\mathrm{R}} \ll 1\right) \\
1 \times 10^{-6} x_{\mathrm{R}} x_{\max , \mathrm{R}}^{5} & \left(1 \ll x_{\mathrm{R}} \lesssim x_{\max , \mathrm{R}}\right) \\
7 \times 10^{-7} x_{\mathrm{R}}^{7} & \left(x_{\max , \mathrm{R}}^{5 / 6} x_{\mathrm{R}} \lesssim x_{\max , \mathrm{R}}\right) \\
(\operatorname{sharp~drop}) & \left(x_{\max , \mathrm{R}} \lesssim x_{\mathrm{R}} \leq 2 x_{\max , \mathrm{R}}\right)
\end{array},\right.
$$

neglecting a logarithmic factor for the second line.

\section{DETERMINATION OF REHEATING TEMPERATURE}

In the previous section, we have shown that the induced GWs can be much larger than those previously reported $[10,37,38]$. In the following, we consider the GWs induced by the almost scale-invariant power spectrum, given in Eq. (16), with $A_{s}=2.1 \times 10^{-9}, k_{*}=0.05 \mathrm{Mpc}^{-1}$, and $n_{s}=0.96$ [44]. Figure 2 shows the sensitivity curves of current and future GW experiments and plots for $\Omega_{\mathrm{GW}}$ of the GWs induced by this power spectrum with $k_{\max }=10^{14} \mathrm{Mpc}^{-1}$. This figure shows that the induced GWs associated with a sudden transition from an eMD era to the RD era could in principle be observable by future projects. Since the height and scale of the peak are determined by the scale of the reheating and the cutoff $k_{\max }$, we discuss what range of the reheating tempera- 


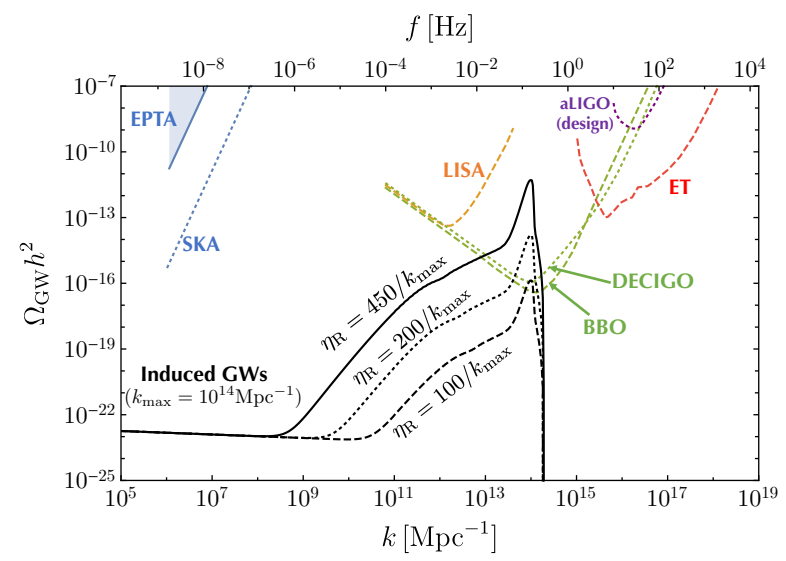

FIG. 2. Effective sensitivities to stochastic GWs of current and future experiments. Note that we plot $\Omega_{\mathrm{GW}, \mathrm{eff}} h^{2} / \sqrt{T_{\mathrm{obs}} f / 10}$ as a sensitivity curve for each experiment. We consider the same experiments and take the same parameters as in Ref. [16]. In particular, we take the same parameter sets of observation time for each experiment: $T_{\text {obs }}=18$ years for EPTA, $T_{\text {obs }}=20$ years for SKA, and $T_{\text {obs }}=1$ year for the other experiments. The shaded regions have already been excluded by the existing observational data. See Ref. [16] for more details about the sensitivity curve of each experiment. Black lines show the energy density parameters of the GWs induced by the power spectrum of $\mathcal{P}_{\zeta}(k)=2.1 \times 10^{-9}\left(k / 0.05 \mathrm{Mpc}^{-1}\right)^{-0.04} \Theta\left(k_{\max }-k\right)$. We take $k_{\max }=10^{14} \mathrm{Mpc}^{-1}$ for all these three lines and $\eta_{\mathrm{R}}=450 / k_{\max }, \eta_{\mathrm{R}}=200 / k_{\max }$ and $\eta_{\mathrm{R}}=100 / k_{\max }$ for each line, respectively.

ture could be probed by future observations searching for GWs.

We adopt an analysis similar to that in our previous paper [16] (see also Ref. [50]). We use the signal-to-noise ratio $\rho$ for $\mathrm{GW}$ interferometers given by [50].

$$
\rho=\sqrt{2 T_{\mathrm{obs}}}\left[\int_{f_{\min }}^{f_{\max }} \mathrm{d} f\left(\frac{\Omega_{\mathrm{GW}}(f)}{\Omega_{\mathrm{GW}, \mathrm{eff}}(f)}\right)^{2}\right]^{1 / 2} .
$$

Here, $T_{\text {obs }}$ is the observation time, and $\left(f_{\min }, f_{\max }\right)$ is the range of observable frequencies for each project. $\Omega_{\mathrm{GW} \text {,eff }}$ is the effective sensitivity curve, which is calculated for each project (see Ref. [16] for detail). For pulsar timing array (PTA) observations, we use [51-53]

$$
\begin{aligned}
\rho= & \sqrt{2 T_{\mathrm{obs}}}\left(\sum_{I=1}^{M} \sum_{J>I}^{M} \chi_{I J}^{2}\right)^{1 / 2} \\
& \times\left[\int_{f_{\min }}^{f_{\max }} \mathrm{d} f\left(\frac{\Omega_{\mathrm{GW}}(f)}{\Omega_{\mathrm{n}}(f)+\Omega_{\mathrm{GW}}(f)}\right)^{2}\right]^{1 / 2} .
\end{aligned}
$$

In this expression, $M$ is the number of the observed pulsars, $\chi_{I J}$ is the Hellings and Downs coefficient, and $\Omega_{\mathrm{n}}$ is the energy density parameter for noise of each pulsar. We take the same parameters and noise power spectrum

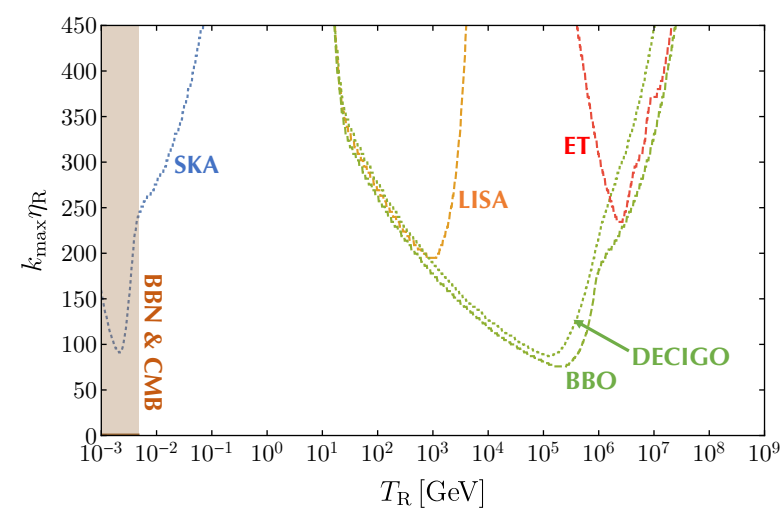

FIG. 3. Relation between the cutoff scale multiplied by $\eta_{\mathrm{R}}$ and the reheating temperature that can be probed by the future observations. We take $T_{\mathrm{obs}}=20 \mathrm{yr}$ for SKA and $T_{\mathrm{obs}}=$ $1 \mathrm{yr}$ for the others. The curves correspond to the values of $k_{\max } \eta_{\mathrm{R}}$ required to reach the signal-to-noise ratio of unity $(\rho=1)$ for the experiments at each reheating temperature. The brown shaded region is already excluded by the big bang nucleosynthesis and the Planck data [55].

as in Ref. [16] assuming that the noise is dominated by the white timing noise [50].

Using the effective sensitivity curves in Fig. 2, we derive the cutoff scale to give $\rho=1$ for each project and reheating temperature. The numerical results are shown in Fig. 3. When we derive the curves, we use the approximation formulas given in Eqs. (B5), (B7), and (B9) to save the computational time. ${ }^{5}$ In this figure, we take $T_{\text {obs }}=20$ years for SKA and $T_{\text {obs }}=1$ year for the other projects and assume no foreground for simplicity. When we obtain these plots, we have used the following relation between the conformal time and the temperature $[16]^{6}$

$$
\begin{gathered}
\frac{a H}{a_{\mathrm{eq}} H_{\mathrm{eq}}}=\frac{1}{\sqrt{2}}\left(\frac{g_{s, \mathrm{eq}}}{g_{s}}\right)^{1 / 3}\left(\frac{g}{g_{\mathrm{eq}}}\right)^{1 / 2} \frac{T}{T_{\mathrm{eq}}} \\
\Rightarrow \frac{\eta_{\mathrm{R}}}{10^{-14} \mathrm{Mpc}}=\left(\frac{g_{s}}{106.75}\right)^{1 / 3}\left(\frac{g}{106.75}\right)^{-1 / 2} \\
\times\left(\frac{T_{\mathrm{R}}}{1.2 \times 10^{7} \mathrm{GeV}}\right)^{-1},
\end{gathered}
$$

\footnotetext{
${ }^{5}$ For simplicity, we use the formulas for the scale-invariant spectrum, given in Eqs. (B5), (B7), and (B9), instead of those for the power-law spectrum, given in Eqs. (B10)-(B13). Since the enhancement of the induced GWs is mainly caused by the perturbations on the smallest scales $\left(k \sim k_{\max }\right)$ and the tilt of the power spectrum is small, we can approximately use the formulas for the scale-invariant spectrum whose amplitude is given by $A_{s}\left(k_{\max } / k_{*}\right)^{n_{s}-1}$. We have also numerically checked that the effect of the tilt with $n_{s}=0.96$ around the smallest scale on the enhanced GW spectrum is negligible.

6 To derive Eq. (21), we use the relation $a_{\mathrm{eq}} H_{\mathrm{eq}}=k_{\mathrm{eq}}(=$ $\left.0.0103 \mathrm{Mpc}^{-1}\right)$ [44, 54]. This relation corrects the factor given in Ref. [16].
} 
where the subscript "eq" means the value at the late matter-radiation equality $(z \sim 3400)$, and $g_{s}$ is the effective degrees of freedom for the entropy density. Note again that the peak scale of the induced GWs corresponds to $k \sim k_{\max }$, not $k \sim 1 / \eta_{\mathrm{R}}$. Figure 3 shows that, in the case of $k_{\max } \eta_{\mathrm{R}}=450$, the ranges of reheating temperature that future observations could investigate are $T_{\mathrm{R}} \lesssim 7 \times 10^{-2} \mathrm{GeV}$ for SKA, $20 \mathrm{GeV} \lesssim T_{\mathrm{R}} \lesssim$ $4 \times 10^{3} \mathrm{GeV}$ for LISA, $20 \mathrm{GeV} \lesssim T_{\mathrm{R}} \lesssim 1 \times 10^{7} \mathrm{GeV}$ for DECIGO, $20 \mathrm{GeV} \lesssim T_{\mathrm{R}} \lesssim 2 \times 10^{7} \mathrm{GeV}$ for $\mathrm{BBO}$, and $4 \times 10^{5} \mathrm{GeV} \lesssim T_{\mathrm{R}} \lesssim 2 \times 10^{7} \mathrm{GeV}$ for ET.

\section{CONCLUSIONS}

We have studied the effects of an eMD era on the induced GWs. In particular, we have focused on a suddenreheating scenario, in which the reheating completes on a timescale much shorter than the Hubble time at that time. Then, we have found that the induced GWs can be significantly enhanced in such a scenario. The main contributions to the enhanced GWs come from the GWs induced during the RD era by the perturbations that entered the horizon during the eMD era. This is due to the fast oscillations of the perturbations after the sudden transition. This enhancement is qualitatively opposite to the suppression of induced GWs in gradual-reheating scenarios, which we report in our accompanying paper [39]. This means that the eMD effects on the induced GWs strongly depend on how the reheating takes place.

We have also numerically calculated the induced GWs with realistic power spectra of curvature perturbations and discussed possibilities of determining the reheating temperature observationally for sudden-reheating scenarios. We have found that if an eMD era lasts for $224 \eta_{\mathrm{eMD} \text {,start }}$, where $\eta_{\mathrm{eMD} \text {,start }}$ is the conformal time at the start of the eMD era, ${ }^{7}$ the reheating temperatures in the range $T_{\mathrm{R}} \lesssim 7 \times 10^{-2} \mathrm{GeV}$ or $20 \mathrm{GeV} \lesssim T_{\mathrm{R}} \lesssim$ $2 \times 10^{7} \mathrm{GeV}$ could be probed by future GW projects, such as SKA, LISA, DECIGO, BBO, and ET. Note that, if an eMD era starts right after inflation era, the duration of the eMD era of $\mathcal{O}(100) \eta_{\mathrm{eMD} \text {,start }}$ corresponds to $\rho_{\text {inf }}^{1 / 4} / T_{\mathrm{R}} \sim \mathcal{O}\left(10^{3}\right)$, where $\rho_{\text {inf }}$ is the energy density during the inflation era.

Since the enhancement of the induced GWs we find is so significant, one may wonder if it is consistent with some physical requirements. Hence, we briefly mention some consistency checks. Let us first discuss energy conservation and backreaction. Note that the dominant part of the energy density of the induced GWs is generated soon after the reheating transition by the shortwavelength modes at around $k \sim k_{\max }$, and the enhance-

\footnotetext{
7 Note that the wavenumber corresponding to the horizon scale at $\eta_{\mathrm{eMD} \text {,start }}$ satisfies $k_{\mathrm{eMD} \text {,start }} \eta_{\mathrm{R}}=450$ because $k_{\mathrm{eMD} \text {,start }}=$ $a_{\mathrm{eMD} \text {,start }} H_{\mathrm{eMD} \text {,start }}=2 / \eta_{\mathrm{eMD} \text {,start }}$ and $\eta_{\mathrm{R}}=225 \eta_{\mathrm{eMD} \text {,start }}$ in this case (the duration is $\eta_{\mathrm{R}}-\eta_{\mathrm{eMD} \text {,start }}=224 \eta_{\mathrm{eMD} \text {,start }}$ ).
}

ment is stronger for $k_{\max }$ closer to the non-linear scale $k_{\mathrm{NL}}$. By definition, the energy density of density perturbations at scales around $k_{\mathrm{NL}}$ is comparable to the energy density of the homogeneous component. On the other hand, in Fig. 1, we have seen that the energy density of the induced GWs is $\Omega_{\mathrm{GW}}\left(\eta_{c}\right) \sim 10^{12} A_{s}^{2} \sim \mathcal{O}\left(10^{-6}\right)$ even for $k_{\max } \eta_{\mathrm{R}}=450\left(k_{\max } \sim k_{\mathrm{NL}}\right)$. The smallness of $\Omega_{\mathrm{GW}}$ implies that the energy density of the induced GWs is much smaller than both the energy density of its source, namely the density perturbations, and the homogeneous component. Thus, a backreaction of the GW production to the thermal history of the Universe would be negligible.

Another concern may be whether or not GWs induced at third order in scalar perturbations are negligible, given the fact that the second-order contributions have turned out to be significant. In other words, one might wonder whether or not sources coming from third order scalar perturbations appear with many derivatives, which can be larger than the dominant source in second order scalar perturbations, $\mathcal{H}^{-2} \Phi^{\prime} \Phi^{\prime}$, even when the perturbations are linear $\left(k \eta_{\mathrm{R}}\right)^{2} \Phi \lesssim 1$. Complete evaluations of the third-order contributions are much more complicated than those for the second-order analysis. Thus, we estimate their orders of magnitudes by listing up possible third-order terms that appear in the evolution equation for tensor perturbations and are consistent with general covariance and the transverse traceless condition. Most of them turned out to be negligible provided that we are in the linear regime, where the density perturbations are less than unity. However, we need to carefully evaluate the contributions involving the first-order scalar perturbations multiplied by the second-order vector perturbations, which are sourced by first-order scalar perturbations at second order, similarly to the induced GWs we have studied. That is because the power spectrum of second-order vector perturbations is larger than that of second-order tensor perturbations [56]. Full evaluations of these contributions are beyond the scope of this work, but we expect that the third-order GWs can be subdominant as long as $k_{\max } \ll k_{\mathrm{NL}}$.

In this paper, we do not take into account the gauge dependence of the induced GWs [43], GW foregrounds due to other astrophysical as well as cosmological sources, and the possible contributions from non-linear perturbations [48, 49], which can arise depending on the length of the eMD era and the amplitude of the small-scale primordial fluctuations. Although these issues remain to be investigated, our result shows that observations of GWs could possibly reveal the reheating history of the Universe in the near future.

\section{ACKNOWLEDGMENTS}

KI and TN thank KEK, Johns Hopkins University and Research Center for the Early Universe, University of Tokyo, for hospitality received during this work. 
KI acknowledges Tomohiro Fujita, Teruaki Suyama, and Masahide Yamaguchi for useful comments. We thank Kai Murai for pointing out the necessity of the fudge factor $Y$ in Eq. (B7). (This factor was accidentally already included in our plots in the previous versions.) This work was supported in part by World Premier International Research Center Initiative (WPI Initiative), MEXT, Japan, the JSPS Research Fellowship for Young Scientists (KI and TT), JSPS KAKENHI Grants No. JP18J12728 (KI), No. JP17H01131 (KK), and No. JP17J00731 (TT), MEXT KAKENHI Grants No. JP15H05889 (KK), No. JP18H04594 (KK) and No. JP19H05114 (KK), and Advanced Leading Graduate Course for Photon Science (KI).

\section{Appendix A: A model that realizes a sudden-reheating transition}

In this Appendix, we build a concrete model in which the reheating happens in a timescale much shorter than the Hubble time at that time. This ensures that $\Phi$ does not decay during the reheating transition, leading to the enhancement of induced GWs, reported in this paper, in contrast to the suppression of induced GWs for a gradual transition, reported in our accompanying paper [39].

Our idea for a sudden reheating is to initially block the decay of the field $\phi$, dominating the energy density in the eMD era, into relativistic daughter particles, collectively denoted by $\chi$, for some reason related to kinematics or symmetry, and then to remove the cause of the blocking in a dynamical manner. For this purpose, we introduce a field $\tau$, which dynamically triggers the decay of $\phi$ into $\chi$. We dub such a field $\tau$ 'triggeron'. In the models we discuss below, the mass of $\chi$ is dependent on the field value of $\tau$ and a quick change of that field value causes a sudden decay of $\phi$ to $\chi$, which we identify as a sudden reheating.

\section{A scenario for a sudden reheating triggered by a fast rolling field}

The main ideas of this model are as follows. At first, the initial triggeron value is sufficiently large so that the decay of $\phi$ into two $\chi$ particles is kinematically forbidden. When the Hubble parameter becomes comparable to the triggeron mass $m$, the triggeron starts to roll down its potential quickly, and it passes through some critical value at which the decay channel of $\phi$ opens. If the decay rate is much larger than the Hubble scale, the reheating transition completes quickly.

We consider a simple model that involves three canonically normalized real scalar fields $\phi, \tau$, and $\chi$ to demonstrate the ideas. One can easily generalize this model by considering e.g. complex scalar fields, fermions, or gauge bosons. The Lagrangian density we assume is

$$
\begin{aligned}
& \mathcal{L}=-\frac{1}{2} \partial^{\mu} \phi \partial_{\mu} \phi-\frac{1}{2} \partial^{\mu} \chi \partial_{\mu} \chi-\frac{1}{2} \partial^{\mu} \tau \partial_{\mu} \tau-V, \\
& V=\frac{1}{2} M^{2} \phi^{2}+\frac{1}{2} m^{2} \tau^{2}+\frac{\lambda}{4} \tau^{2} \chi^{2}+\frac{c}{2} M \phi \chi^{2},
\end{aligned}
$$

where $M$ and $m$ denote the masses of $\phi$ and $\tau$, respectively, satisfying $M^{2} \gg m^{2}$, and $\lambda$ and $c$ are dimensionless coupling constants. The third term in the potential can be interpreted as the $\tau$-dependent mass term for $\chi$, and the last term provides the decay channel of $\phi$ into $2 \chi$ particles, once the decay becomes kinematically allowed. The decay rate of $\phi$ into $2 \chi$ particles is

$$
\Gamma=\frac{c^{2} M}{32 \pi} \sqrt{1-\frac{m_{\chi, \mathrm{eff}}^{2}}{(M / 2)^{2}}} \Theta\left(M^{2}-4 m_{\chi, \mathrm{eff}}^{2}\right)
$$

where $m_{\chi, \text { eff }}^{2}=\left\langle\lambda \tau^{2} / 2\right\rangle$ is the effective mass squared of $\chi$ and it is determined by the time-dependent expectation value of $\tau$, as mentioned above. Note that the decay rate is non-zero only when the decay is kinematically possible, i.e., $m_{\chi, \text { eff }}<M / 2$, otherwise, it vanishes. The critical value of triggeron field at which the decay channel opens is $\tau_{\mathrm{c}}=M / \sqrt{2 \lambda}{ }^{8}$

There are some conditions for this scenario to work. Obviously, the initial field value of triggeron $\tau_{0}$ should be large enough to satisfy $\tau_{0}>\tau_{\mathrm{c}}$. (We may assume $\tau_{0} \geq 0$ without loss of generality.) To make the reheating transition quick, the speed of $\tau$ needs to be sufficiently large when it passes through the critical point $\tau_{\mathrm{c}}$, hence we assume $\tau_{0} \gg \tau_{\mathrm{c}}$. On the other hand, the triggeron field should not dominate the energy density, and so $\tau_{0}$ should be much less than the reduced Planck mass $M_{\mathrm{P}}$. Thus, the required condition for $\tau_{0}$ is

$$
\tau_{\mathrm{c}} \ll \tau_{0} \ll M_{\mathrm{P}} .
$$

Second, once the decay becomes kinematically possible, the typical decay rate should be much larger than the Hubble scale, $\Gamma \gg H$. This requires $c^{2} M \gg m$. We also assume that $\tau$ eventually decays into radiation before it would dominate the energy density.

\footnotetext{
8 To follow the evolutions of the mass of the daughter particles $\chi$ after the decay of $\phi$, we need to take into account the backreaction of the particle production effect to the dynamics of $\tau$, whose dedicated analysis is beyond the scope of this paper. Once most of the energy density in $\phi$ has been converted to a large number of $\chi$ particles when they are almost massless, energy conservation implies that $\tau$ cannot move significantly. A similar backreaction effect is discussed in the context of preheating [57]. We expect that $\tau$ is trapped around the origin and assume that the daughter particles $\chi$ remain relativistic in the following analyses. Even if the daughter particles do not behave as relativistic particles due to its varying mass, the sudden reheating is realized in the case where the daughter particles decay or annihilate to other light particles, including the Standard Model particles, within a timescale much shorter than the Hubble time at that time.
} 


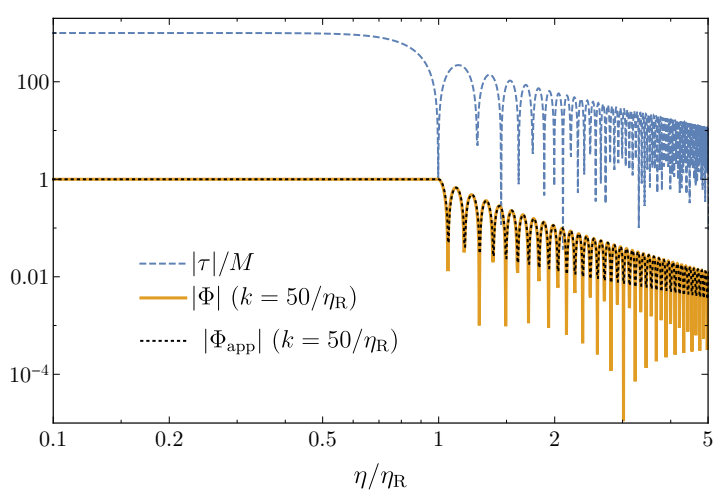

FIG. 4. Numerical results for the evolutions of the gravitational potential $\Phi$ and the triggeron $\tau$, normalized by $M$. We also plot the analytical formula of $\Phi$, given by Eq. (7), with the black dotted line. We take $\lambda=0.1, c=0.1$, and $\tau_{0}=1000 M$.

Let us present the time evolution of the gravitational potential $\Phi$ to show how this model works. Figure 4 shows the evolution of $\Phi$ in addition to that of the field $\tau$. For $\Phi$, we use the equations for perturbations that are used in our accompanying paper [39] to take into account the decay of $\phi$ to $\chi$. This figure shows that the analytical expression of $\Phi$, given in Eq. (8), is satisfactorily accurate in sudden-reheating scenarios.

Since $\phi$ and $\tau$ are independent degrees of freedom, fluctuations in $\tau$ will introduce additional curvature perturbations and non-Gaussianity due to the modulated reheating mechanism [58-62]. To estimate those quantities, let us first note that the time evolution of the triggeron is given by $\tau=\tau_{0} \sin (m t) /(m t)$. The time when it reaches the minimum $(\tau=0)$ is $m t=\pi$, but it reaches the critical value slightly before. The decay time is thus estimated to be

$$
m t=\pi\left(1-\frac{\tau_{c}}{\tau_{0}}\right)
$$

As discussed e.g. in Ref. [63], the e-folding number is related to the decay time as

$$
e^{N} \propto t^{1 / 6}
$$

Thus, we can calculate $N^{\prime}=(1 / 6) t^{\prime} / t$ and $N^{\prime \prime}=$ $(1 / 6)\left(t^{\prime \prime} / t-\left(t^{\prime} / t\right)^{2}\right)$ where the prime denotes differentiation with respect to $\tau_{0}$, and $t$ is evaluated at the decay time. Explicitly,

$$
N^{\prime} \simeq \frac{\tau_{\mathrm{c}}}{6 \tau_{0}^{2}}, \quad \quad N^{\prime \prime} \simeq-\frac{\tau_{\mathrm{c}}}{3 \tau_{0}^{3}},
$$

noting $\tau_{c} \ll \tau_{0}$. Using these values, we obtain

$$
\begin{aligned}
\mathcal{P}_{\zeta^{(\tau)}} & =\left(N^{\prime} \delta \tau_{0}\right)^{2} \simeq \frac{1}{36}\left(\frac{\tau_{c}}{\tau_{0}}\right)^{2}\left(\frac{H_{\mathrm{inf}}}{2 \pi \tau_{0}}\right)^{2}, \\
f_{\mathrm{NL}} & =\frac{5}{6}\left(\frac{\mathcal{P}_{\zeta^{(\tau)}}}{\mathcal{P}_{\zeta}}\right)^{2} \frac{N^{\prime \prime}}{\left(N^{\prime}\right)^{2}} \simeq-10\left(\frac{\mathcal{P}_{\zeta^{(\tau)}}}{\mathcal{P}_{\zeta}}\right)^{2} \frac{\tau_{0}}{\tau_{c}},
\end{aligned}
$$

where $\zeta$ represents the total curvature perturbation, and $\zeta^{(\tau)}$ is the contribution to $\zeta$ from $\tau$. Note that $f_{\mathrm{NL}}$ appears to contain a large factor $\tau_{0} / \tau_{c}$, but the above expression implicitly contains the inverse of this factor with a higher power. Hence, $f_{\mathrm{NL}}$ can be sufficiently small. We conclude that non-Gaussianity can be small enough to be consistent with observations provided that $\tau_{0} \gg \tau_{\mathrm{c}}$ is satisfied.

Let us interpret the above model. It is quite natural that the decay of a field is prohibited by some symmetry. For example, the lightest particle charged under some unbroken symmetry is absolutely stable. This is usually applied to dark matter model building to explain its stability [64]. Thus, we assume that $\phi$ is charged under some symmetry. If the scalar field is real, as in the above toy model, the possible charge assignment is limited, and so the scalar fields will be complex in a more realistic situation. In this context, $\tau$ must be assumed to be a singlet (non-charged) with respect to the symmetry that protects $\phi$ 's stability because otherwise its initial nonzero expectation value spontaneously breaks the symmetry. $\chi$ can be interpreted as some charged particle, initially heavier than $\phi$ due to its $\tau$-dependent mass. However, it subsequently becomes lighter than $\phi$, which triggers the $\phi$ decay. For example, we can assign $\phi$ charge +2 and $\chi$ charge -1 . Or, we can assign $\phi$ and $\chi$ the same charge and introduce a chargeless field $\chi^{\prime}$ with an interaction such as $\phi \chi^{\dagger} \chi^{\prime}$. In this way, various generalizations of our simple model would be possible. The produced relativistic $\chi$ particles and antiparticles are assumed to produce a thermal bath containing Standard Model particles through scattering and annihilation, which reheats the Universe.

Alternatively, we may interpret $\tau$ as some symmetry breaking field. When a symmetry is broken, it is often the case that charged fields (corresponding to $\chi$ ) become massive. For example, the Higgs mechanism makes gauge bosons massive. In the Standard Model, it also makes fermions massive through Yukawa interactions. One of the flat directions in the minimal supersymmetric standard model [65] would be a good candidate for this purpose since most of the fields in the theory (corresponding to $\chi$ ) can be massive when it obtains a finite expectation value. In this case, all the possible decay channels of $\phi$ must be kinematically blocked or sufficiently suppressed.

\section{Another sudden-reheating scenario realized by a field that experiences a first order phase transition}

Suppose that $\phi$ is protected by a symmetry from decaying, without any decay channels of $\phi$ to lighter particles. Let us further assume that $\tau$ is charged under the symmetry and is too heavy for $\phi$ to decay into. There may be an interaction term of the form

$$
\mathcal{L}=c \tau \phi \chi \chi+\ldots
$$


where $c$ is a coupling constant. Suppose that initially the field value of $\tau$ is zero, to be contrasted with the previous model. Then the decay of $\phi$ becomes possible once $\tau$ acquires a finite vacuum expectation value, thereby spontaneously breaking the symmetry.

Such a symmetry-breaking phase transition can occur suddenly if the phase transition is first order. The transition occurs through the tunneling effect, and the tunneling rate is exponentially sensitive to the cosmic temperature (to be more precise, the temperature of the thermal bath to which $\tau$ is coupled), and hence such a transition is sudden [66]. After the transition, $\phi$ becomes able to decay into $\chi$ particles. Provided that this decay rate is much larger than the Hubble parameter, the decay completes within a timescale much shorter than the Hubble time at that time. Associated with the decay of $\phi$, the temperature increases, which may restore the symmetry temporarily. Thus, the importance of the backreaction to the decay of $\phi$ requires a further study. Eventually, the temperature decreases, and $\tau$ settles to the symmetrybreaking vacuum.

One way to suppress the backreaction may be to assume that the initial thermal bath is made up of a hidden sector with $\tau$ being a portal to the visible sector. Then, the increase in the temperature felt by $\tau$ would not be significantly affected by the decay of $\phi$.

\section{Appendix B: Approximate analytic formulas for induced GWs}

Here, we derive analytic formulas of the spectrum of induced GWs with some approximations based on suddenreheating scenarios. During an eMD era, $\Phi$ is constant, and in the RD era, after reheating, the general solution of $\Phi$ is given by the sum involving spherical Bessel functions. These solutions for the two epochs are connected at the transition.

As we can see in Fig. 4, $\Phi$ can be well approximated by Eq. (8) in sudden-reheating scenarios. The explicit forms of the coefficients $A$ and $B$ in Eqs. (11) and (12) are

$$
\begin{aligned}
& A\left(x_{\mathrm{R}}\right)=\left(-\frac{x_{\mathrm{R}}^{2}}{36}+1\right) \cos \frac{x_{\mathrm{R}}}{2 \sqrt{3}}+\frac{\sqrt{3}}{6} x_{\mathrm{R}} \sin \frac{x_{\mathrm{R}}}{2 \sqrt{3}}, \\
& B\left(x_{\mathrm{R}}\right)=\left(-\frac{x_{\mathrm{R}}^{2}}{36}+1\right) \sin \frac{x_{\mathrm{R}}}{2 \sqrt{3}}-\frac{\sqrt{3}}{6} x_{\mathrm{R}} \cos \frac{x_{\mathrm{R}}}{2 \sqrt{3}} .
\end{aligned}
$$

Note that if we replace $x-x_{\mathrm{R}} / 2$ in the expression for $\Phi$ by $x$, our formulas become those in the case of a pure $\mathrm{RD}$ era, and hence we can use the general formulas in Appendix A of Ref. [10]. We substitute the above $A$ and $B$ as well as $C=-\cos \left(x-x_{\mathrm{R}} / 2\right)$ and $D=\sin \left(x-x_{\mathrm{R}} / 2\right)$ into these equations with $x_{1}$ and $x_{2}$ replaced by $x_{1}-x_{\mathrm{R}} / 2$ and $x_{2}-x_{\mathrm{R}} / 2$, respectively. The function $I$ is split into two terms as in Eq. (13). The contributions generated during an eMD era have been derived in Ref. [37] and revised in Ref. [10], and so we here mainly discuss the contributions generated during the RD era, $I_{\mathrm{RD}}$. As explained in the main text, this behaves very differently from the counterpart for a pure $\mathrm{RD}$ era, which is obtained in the limit $x_{\mathrm{R}} \rightarrow 0$, because of fast oscillations of the modes that are already inside the horizon at the reheating transition. Extracting the redshift factor from the function, $I_{\mathrm{RD}}=\frac{1}{x-x_{\mathrm{R}} / 2} \mathcal{I}_{\mathrm{RD}}$, we first calculate $\mathcal{I}_{\mathrm{RD}}$, for which we can use the results of Ref. [10].

Below, we use two different approximations to obtain two main contributions. The first approximation is valid for the large-scale modes with $k \ll k_{\max }$, and the second approximation extracts the resonant contributions at $k \lesssim 2 k_{\max } / \sqrt{3}$. The sum of these two contributions turns out to explain the results of numerical integrations well. For simplicity, we first consider the spectrum given in Eq. (16) with $n_{\mathrm{s}}=1$. Generalization to cases with an arbitrary $n_{\mathrm{s}}(>-3 / 2)$ is discussed at the end of this Appendix.

\section{Large-scale approximation}

As long as the scale $k^{-1}$ under consideration is much larger than the smallest scale $k_{\max }^{-1}$, the integrations over $u$ and $v$, wavenumbers in units of $k$, are dominated by the large $t(\equiv u+v-1)$ region $\left(t \sim x_{\max , \mathrm{R}} / x_{\mathrm{R}}\right)$, hence $t x_{\mathrm{R}} \sim$ $x_{\max , \mathrm{R}} \gg 1$. After taking late-time $(x \gg 1)$ oscillation average and changing variables from $u$ and $v$, to $t$ and $s \equiv u-v$, we find

$$
\begin{aligned}
\overline{\mathcal{I}_{\mathrm{RD}}^{2}} \simeq & \frac{9 t^{4} x_{\mathrm{R}}^{8}}{163840000}\left(\pi^{2}+\pi^{2} \cos \frac{s x_{\mathrm{R}}}{\sqrt{3}}+2 \mathrm{Ci}\left(\frac{x_{\mathrm{R}}}{2}-\frac{s x_{\mathrm{R}}}{2 \sqrt{3}}\right)^{2}+2 \mathrm{Ci}\left(\frac{x_{\mathrm{R}}}{2}+\frac{s x_{\mathrm{R}}}{2 \sqrt{3}}\right)^{2}+4 \cos \frac{s x_{\mathrm{R}}}{\sqrt{3}} \mathrm{Ci}\left(\frac{x_{\mathrm{R}}}{2}+\frac{s x_{\mathrm{R}}}{2 \sqrt{3}}\right) \mathrm{Ci}\left(\frac{x_{\mathrm{R}}}{2}-\frac{s x_{\mathrm{R}}}{2 \sqrt{3}}\right)\right. \\
& +2 \pi \sin \frac{s x_{\mathrm{R}}}{\sqrt{3}}\left(\mathrm{Ci}\left(\frac{x_{\mathrm{R}}}{2}+\frac{s x_{\mathrm{R}}}{2 \sqrt{3}}\right)-\mathrm{Ci}\left(\frac{x_{\mathrm{R}}}{2}-\frac{s x_{\mathrm{R}}}{2 \sqrt{3}}\right)\right)-2 \pi\left(1+\cos \frac{s x_{\mathrm{R}}}{\sqrt{3}}\right)\left(\mathrm{Si}\left(\frac{x_{\mathrm{R}}}{2}-\frac{s x_{\mathrm{R}}}{2 \sqrt{3}}\right)+\mathrm{Si}\left(\frac{x_{\mathrm{R}}}{2}+\frac{s x_{\mathrm{R}}}{2 \sqrt{3}}\right)\right) \\
& +4 \sin \frac{s x_{\mathrm{R}}}{\sqrt{3}}\left(\mathrm{Ci}\left(\frac{x_{\mathrm{R}}}{2}-\frac{s x_{\mathrm{R}}}{2 \sqrt{3}}\right) \mathrm{Si}\left(\frac{x_{\mathrm{R}}}{2}+\frac{s x_{\mathrm{R}}}{2 \sqrt{3}}\right)-\mathrm{Ci}\left(\frac{x_{\mathrm{R}}}{2}+\frac{s x_{\mathrm{R}}}{2 \sqrt{3}}\right) \mathrm{Si}\left(\frac{x_{\mathrm{R}}}{2}-\frac{s x_{\mathrm{R}}}{2 \sqrt{3}}\right)\right)
\end{aligned}
$$




$$
\left.+2 \mathrm{Si}\left(\frac{x_{\mathrm{R}}}{2}-\frac{s x_{\mathrm{R}}}{2 \sqrt{3}}\right)^{2}+2 \mathrm{Si}\left(\frac{x_{\mathrm{R}}}{2}+\frac{s x_{\mathrm{R}}}{2 \sqrt{3}}\right)^{2}+4 \cos \frac{s x_{\mathrm{R}}}{\sqrt{3}} \mathrm{Si}\left(\frac{x_{\mathrm{R}}}{2}+\frac{s x_{\mathrm{R}}}{2 \sqrt{3}}\right) \mathrm{Si}\left(\frac{x_{\mathrm{R}}}{2}-\frac{s x_{\mathrm{R}}}{2 \sqrt{3}}\right)\right),
$$

where we have kept only terms with highest powers of $t$. The sine and cosine integrals are defined as $\operatorname{Si}(x)=$ $\int_{0}^{x} \mathrm{~d} z \sin (z) / z$ and $\operatorname{Ci}(x)=-\int_{x}^{\infty} \mathrm{d} z \cos (z) / z$. When we vary $s$, the above quantity varies approximately by a factor of two at most. However, the angular factor (the factor in the first line of Eq. (3)) in the large $t$ limit is $\left(s^{2}-1\right)^{2}$, which suppresses the nonzero $s$ part, and so it turns out that setting $s=0$ is a good approximation for calculating $\Omega_{\mathrm{GW}}$ with $10 \%$ errors at most. If we set $s=0$, it is simplified as

$$
{\overline{\mathcal{I}_{\mathrm{RD}}^{2}}}_{s=0} \simeq \frac{9 t^{4} x_{\mathrm{R}}^{8}\left(4 \mathrm{Ci}\left(\frac{x_{\mathrm{R}}}{2}\right)^{2}+\left(\pi-2 \mathrm{Si}\left(\frac{x_{\mathrm{R}}}{2}\right)\right)^{2}\right)}{81920000} .
$$

This expression is so simple that we can analytically integrate it over $t$ and $s$. The integration region is $0 \leq s \leq 1$ and $0 \leq t \leq-s+2 \frac{x_{\max , \mathrm{R}}}{x_{\mathrm{R}}}-1$ for $x_{\mathrm{R}} \leq x_{\max , \mathrm{R}}$, and $0 \leq s \leq 2 \frac{x_{\max , \mathrm{R}}}{x_{\mathrm{R}}}-1$ and $0 \leq t \leq-s+2 \frac{x_{\max , \mathrm{R}}}{x_{\mathrm{R}}}-1$ for $x_{\mathrm{R}}>x_{\max , \mathrm{R}}$. For each case, there is also an integration region obtained by the replacement $s \rightarrow-s$, but the symmetry under this inversion ensures that the total result is obtained by doubling the result obtained from the integration region with $s>0$. Then the GW spectrum under the large-scale (LS) approximation is

$$
\begin{aligned}
\Omega_{\mathrm{GW}, \mathrm{RD}}^{(\mathrm{LS})}\left(\eta_{c}, k\right) \simeq & \frac{4 \mathrm{Ci}\left(\frac{x_{\mathrm{R}}}{2}\right)^{2}+\left(\pi-2 \operatorname{Si}\left(\frac{x_{\mathrm{R}}}{2}\right)\right)^{2}}{86016000000} A_{\mathrm{s}}^{2} x_{\mathrm{R}}^{3} x_{\max , \mathrm{R}}^{5} \times \\
& \left(\Theta\left(x_{\max , \mathrm{R}}-x_{\mathrm{R}}\right)\left(5376-17640 \widetilde{k}+23760 \widetilde{k}^{2}-16425 \widetilde{k}^{3}+5825 \widetilde{k}^{4}-847 \widetilde{k}^{5}\right)\right. \\
& \left.+\Theta\left(x_{\mathrm{R}}-x_{\max , \mathrm{R}}\right) \widetilde{k}^{-5}(2-\widetilde{k})^{6}\left(4-8 \widetilde{k}-9 \widetilde{k}^{2}+13 \widetilde{k}^{3}+49 \widetilde{k}^{4}\right)\right),
\end{aligned}
$$

where $\widetilde{k}=x_{\mathrm{R}} / x_{\max , \mathrm{R}}=k / k_{\max }$.

\section{The resonant peak contributions}

It seems challenging to obtain a simple expression for the contributions from the $x_{\mathrm{R}} \simeq x_{\max , \mathrm{R}}$ region. This is partly because the leading-order terms (with the highest power of $x_{\mathrm{R}}$ ) cancel for generic values of $t$ and $s$, and the next-to-leading order terms are complicated.

Let us focus on a specific contribution that corresponds to the resonance-like peak (logarithmic divergence) at $t=\sqrt{3}-1$ in the case of the monochromatic source in a pure $\mathrm{RD}$ era [4]. The origin of the peak is the limit $x_{\mathrm{R}} \rightarrow 0$ of the $\mathrm{Ci}$ function. In the present case, we do not take the limit $x_{\mathrm{R}} \rightarrow 0$, but instead we focus on contributions from the region where the integration variable $t$ hits the logarithmic singularity of the function $\mathrm{Ci}$, possibly causing an enhancement. For this purpose, we do not take the large $t$ limit. Instead, we can take the large $x_{\mathrm{R}}$ limit since it turns out that this effect is most efficient for the smallest-scale modes.

We focus on the terms containing the $\mathrm{Ci}$ function whose argument can vanish, neglecting the other terms.
Furthermore, we take the late time limit $x \rightarrow \infty$ as well as oscillation average. With these approximations, we find

$$
\overline{\mathcal{I}_{\mathrm{RD}}^{2}} \approx Y \frac{9\left(-5+s^{2}+2 t+t^{2}\right)^{4} x_{\mathrm{R}}^{8}}{81920000(1-s+t)^{2}(1+s+t)^{2}} \mathrm{Ci}(|y|)^{2}
$$

where $y \equiv(t-\sqrt{3}+1) x_{\mathrm{R}} /(2 \sqrt{3})$ and $Y$ is a fudge factor of order unity. We focus on spiky contributions around $y=0$ or equivalently $t=\sqrt{3}-1$. Except for the argument of the Ci function, we may set $t=\sqrt{3}-1$, which enables us to do the integration over $s$. Then, the resonant contribution to the GW spectrum is

$$
\begin{aligned}
\Omega_{\mathrm{GW}, \mathrm{RD}}^{(\mathrm{res})}\left(\eta_{c}, k\right) \simeq & Y \int_{-s_{0}\left(x_{\mathrm{R}}\right)}^{s_{0}\left(x_{\mathrm{R}}\right)} \mathrm{d} s \frac{3\left(1-s^{2}\right)^{2}}{81920000} A_{\mathrm{s}}^{2} x_{\mathrm{R}}^{8} \\
& \times 2 \int_{0}^{1} \mathrm{~d} y \operatorname{Ci}(y)^{2} \frac{2 \sqrt{3}}{x_{\mathrm{R}}} \\
= & Y \frac{2.30285}{102400000} \sqrt{3} A_{\mathrm{s}}^{2} x_{\mathrm{R}}^{7} s_{0}\left(x_{\mathrm{R}}\right) \\
& \times\left(15-10 s_{0}^{2}\left(x_{\mathrm{R}}\right)+3 s_{0}^{4}\left(x_{\mathrm{R}}\right)\right),
\end{aligned}
$$




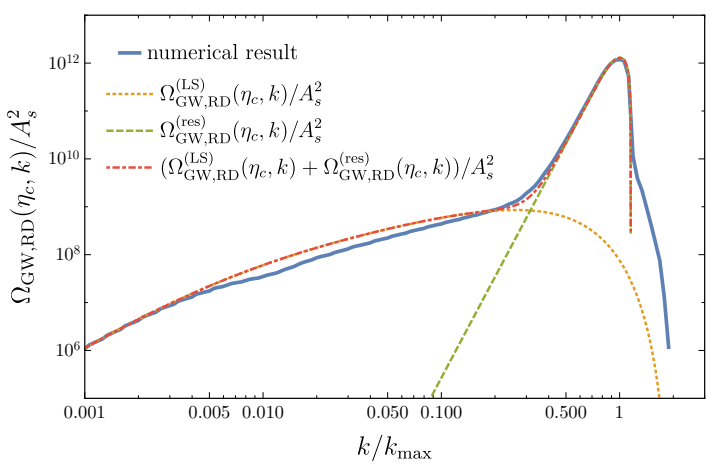

FIG. 5. Comparison of the analytic and numerical results for the induced GWs. The blue solid line shows the numerical result. The orange dotted, greed dashed, and red dotdashed lines show the large-scale approximation [Eq. (B5)], the resonant contribution [Eq. (B7)], and their sum, respectively. We take the power spectrum given in Eq. (16) with $k_{\max }=450 / \eta_{\mathrm{R}}$ and $n_{s}=1$.

where

$$
s_{0}\left(x_{\mathrm{R}}\right)= \begin{cases}1 & x_{\mathrm{R}} \leq \frac{2 x_{\max , \mathrm{R}}}{1+\sqrt{3}} \\ 2 \frac{x_{\max , \mathrm{R}}}{x_{\mathrm{R}}}-\sqrt{3} & \frac{2 x_{\max }, \mathrm{R}}{1+\sqrt{3}} \leq x_{\mathrm{R}} \leq \frac{2 x_{\max , \mathrm{R}}}{\sqrt{3}} \\ 0 & \frac{2 x_{\max , \mathrm{R}}}{\sqrt{3}} \leq x_{\mathrm{R}}\end{cases}
$$

In the first equality in Eq. (B7), we have changed the integration variable from $t$ to $y$ with the Jacobian factor $2 \sqrt{3} / x_{\mathrm{R}}$. This integration is for the spiky part, and so we limit the integration region to $|y|<1$. The choice of the integration boundary here is somewhat arbitrary, but this uncertainty can also be absorbed by the fudge factor $Y$. We determine the value of $Y$ by comparing Eq. (B7) with the numerical result. We find that $Y=2.3$ is a good fit, so we set $Y=2.3$ throughout this work.

The total spectrum is approximated by the contribution produced after the reheating transition, $\Omega_{\mathrm{GW}} \simeq$ $\Omega_{\mathrm{GW}, \mathrm{RD}}$, which is given by the sum of Eqs. (B5) and (B7):

$$
\Omega_{\mathrm{GW}, \mathrm{RD}} \simeq \Omega_{\mathrm{GW}, \mathrm{RD}}^{(\mathrm{LS})}+\Omega_{\mathrm{GW}, \mathrm{RD}}^{(\mathrm{res})} .
$$

This is compared with the numerical result in Fig. 5 . From this figure, we can see that those approximate analytic formulas fit the numerical result very well.

The $k$ dependence of $\Omega_{\mathrm{GW}}$ is summarized as follows. It is proportional to $k^{3}$, neglecting a logarithmic factor, for $k \lesssim 1 / \eta_{\mathrm{R}}$, then it scales as $k$ for $k \gtrsim 1 / \eta_{\mathrm{R}}$. The slope of the resonant contribution is $k^{7}$, which peaks at $k \simeq 2 k_{\max } / \sqrt{3}$. Finally, it decrease sharply, and vanishes at $k=2 k_{\text {max }}$. This behavior is summarized in Eq. (18).

\section{Approximate analytic formulas for induced GWs from power-law primordial spectra}

We can generalize our calculations to power-law primordial spectra with a cutoff [see Eq. (16)]. We can use the formulas of $\overline{\mathcal{I}_{\mathrm{RD}}^{2}}$ obtained above. Using the large $t$ approximation and assuming $n_{\mathrm{s}}>-3 / 2$, we obtain the following expression:

$$
\begin{aligned}
\Omega_{\mathrm{GW}, \mathrm{RD}}^{(\mathrm{LS})} \simeq \frac{3\left(4 \mathrm{Ci}\left(\frac{x_{R}}{2}\right)^{2}+\left(\pi-2 \mathrm{Si}\left(\frac{x_{R}}{2}\right)\right)^{2}\right) A_{\mathrm{s}}^{2} x_{\max , \mathrm{R}}^{8}}{2^{17+2 n_{\mathrm{s}}} \times 625\left(3+2 n_{\mathrm{s}}\right)}\left(\frac{2 x_{\max , \mathrm{R}}}{x_{\mathrm{R}}}-1\right)^{2 n_{\mathrm{s}}}\left(\frac{x_{\mathrm{R}}}{x_{*, \mathrm{R}}}\right)^{2\left(n_{s}-1\right)} \\
\times\left(\widetilde{\Omega}_{\mathrm{GW}, \mathrm{RD}}^{(\mathrm{LS}, 1)} \Theta\left(x_{\max , \mathrm{R}}-x_{\mathrm{R}}\right)+\widetilde{\Omega}_{\mathrm{GW}, \mathrm{RD}}^{(\mathrm{LS}, 2)} \Theta\left(x_{\mathrm{R}}-x_{\max , \mathrm{R}}\right)\right) \Theta\left(2 x_{\max , \mathrm{R}}-x_{\mathrm{R}}\right),
\end{aligned}
$$

where

$$
\begin{aligned}
\widetilde{\Omega}_{\mathrm{GW}, \mathrm{RD}}^{(\mathrm{LS}, 1)}= & \frac{1}{\left(2+n_{\mathrm{s}}\right)\left(3+n_{\mathrm{s}}\right)\left(4+n_{\mathrm{s}}\right)\left(5+2 n_{\mathrm{s}}\right)\left(7+2 n_{\mathrm{s}}\right)} \times\left(1536-6144 \widetilde{k}+\left(7168-1920 n_{\mathrm{s}}-256 n_{\mathrm{s}}^{2}\right) \widetilde{k}^{2}\right. \\
& +\left(5760 n_{\mathrm{s}}+768 n_{\mathrm{s}}^{2}\right) \widetilde{k}^{3}+\left(1328 n_{\mathrm{s}}+3056 n_{\mathrm{s}}^{2}+832 n_{\mathrm{s}}^{3}+64 n_{\mathrm{s}}^{4}\right) \widetilde{k}^{4} \\
& -\left(7168+12256 n_{\mathrm{s}}+7392 n_{\mathrm{s}}^{2}+1664 n_{\mathrm{s}}^{3}+128 n_{\mathrm{s}}^{4}\right) \widetilde{k}^{5}+\left(7392+10992 n_{\mathrm{s}}+5784 n_{\mathrm{s}}^{2}+1248 n_{\mathrm{s}}^{3}+96 n_{\mathrm{s}}^{4}\right) \widetilde{k}^{6} \\
& -\left(2784+3904 n_{\mathrm{s}}+1960 n_{\mathrm{s}}^{2}+416 n_{\mathrm{s}}^{3}+32 n_{\mathrm{s}}^{4}\right) \widetilde{k}^{7}+\left(370+503 n_{\mathrm{s}}+247 n_{\mathrm{s}}^{2}+52 n_{\mathrm{s}}^{3}+4 n_{\mathrm{s}}^{4} \widetilde{k}^{8}\right. \\
& \left.-256(1-\widetilde{k})^{6}\left(6+6\left(2+n_{\mathrm{s}}\right) \widetilde{k}+\left(2+n_{\mathrm{s}}\right)\left(5+2 n_{\mathrm{s}}\right) \widetilde{k}^{2}\right)\left(1-\frac{\widetilde{k}}{2-\widetilde{k}}\right)^{2 n_{\mathrm{s}}}\right) \\
\widetilde{\Omega}_{\mathrm{GW}, \mathrm{RD}}^{(\mathrm{LS}, 2)}= & 2(2-\widetilde{k})^{4} \Gamma\left(4+2 n_{\mathrm{s}}\right)\left(\frac{\widetilde{k}^{4}}{\Gamma\left(5+2 n_{\mathrm{s}}\right)}-\frac{4 \widetilde{k}^{2}(2-\widetilde{k})^{2}}{\Gamma\left(7+2 n_{\mathrm{s}}\right)}+\frac{24(2-\widetilde{k})^{4}}{\Gamma\left(9+2 n_{\mathrm{s}}\right)}\right)
\end{aligned}
$$


with $\Gamma(x)$ denoting the Gamma function. The other important component, the resonance contribution, is obtained as

$$
\begin{aligned}
\Omega_{\mathrm{GW}, \mathrm{RD}}^{(\mathrm{res})}= & Y \frac{2.30285 \times \sqrt{3} 3^{n_{\mathrm{s}}}}{2^{13+2 n_{\mathrm{s}}} \times 625} x_{\mathrm{R}}^{7}\left(\frac{x_{\mathrm{R}}}{x_{*}}\right)^{2\left(n_{\mathrm{s}}-1\right)} s_{0}\left(x_{\mathrm{R}}\right) \\
& \times\left(4_{2} F_{1}\left(\frac{1}{2}, 1-n_{\mathrm{s}} ; \frac{3}{2} ; \frac{s_{0}^{2}\left(x_{\mathrm{R}}\right)}{3}\right)-3_{2} F_{1}\left(\frac{1}{2},-n_{\mathrm{s}} ; \frac{3}{2} ; \frac{s_{0}^{2}\left(x_{\mathrm{R}}\right)}{3}\right)-s_{0}^{2}\left(x_{\mathrm{R}}\right)_{2} F_{1}\left(\frac{3}{2},-n_{\mathrm{s}} ; \frac{5}{2} ; \frac{s_{0}^{2}\left(x_{\mathrm{R}}\right)}{3}\right)\right),
\end{aligned}
$$

where ${ }_{2} F_{1}(a, b ; c ; z)$ is the hypergeometric function, and $s_{0}\left(x_{\mathrm{R}}\right)$ is defined in Eq. (B8). The total spectrum is again approximated by the sum: $\Omega_{\mathrm{GW}} \simeq \Omega_{\mathrm{GW}, \mathrm{RD}}^{(\mathrm{LS})}+\Omega_{\mathrm{GW}, \mathrm{RD}}^{(\mathrm{res})}$.

For completeness and comparison, we also present the formulas of the component of the induced GWs produced during the eMD era, $\Omega_{\mathrm{GW}, \mathrm{eMD}}$. Such a formula is presented in Ref. [10] for the scale-invariant power spectrum of the curvature perturbations with a cutoff scale $k_{\max }$. We generalize it to the power-law spectrum with a cutoff scale. For this purpose, we use the large $t$ approximation once again.

$$
\begin{aligned}
\Omega_{\mathrm{GW}, \mathrm{eMD}}^{(\mathrm{LS})}\left(\eta_{c}, k\right)= & \frac{3 R\left(x_{\mathrm{R}}\right) A_{\mathrm{s}}^{2}\left(\frac{x_{\mathrm{R}}}{x_{*}, \mathrm{R}}\right)^{2\left(n_{\mathrm{s}}-1\right)} \Theta\left(2 x_{\mathrm{max}, \mathrm{R}}-x_{\mathrm{R}}\right)}{25 \times 2^{1+2 n_{\mathrm{s}}} n_{\mathrm{s}}\left(1+n_{\mathrm{s}}\right)\left(2+n_{\mathrm{s}}\right)\left(-1+2 n_{\mathrm{s}}\right)\left(1+2 n_{\mathrm{s}}\right)\left(3+2 n_{\mathrm{s}}\right) \widetilde{k}^{4+2 n_{\mathrm{s}}}} \times \\
& \left(-4^{2+n_{\mathrm{s}}}(1-\widetilde{k})^{2\left(1+n_{\mathrm{s}}\right)}\left(6+6 n_{\mathrm{s}} \widetilde{k}+n_{\mathrm{s}}\left(1+2 n_{\mathrm{s}} \widetilde{k}^{2}\right) \Theta(1-\widetilde{k})\right.\right. \\
& \left.+(2-\widetilde{k})^{2 n_{\mathrm{s}}}\left(96-192 \widetilde{k}+\left(96-8 n_{\mathrm{s}}\left(7+2 n_{\mathrm{s}}\right)\right) \widetilde{k}^{2}+8 n_{\mathrm{s}}\left(7+2 n_{\mathrm{s}}\right) \widetilde{k}^{3}+n_{\mathrm{s}}\left(1+2 n_{\mathrm{s}}\right)\left(11+n_{\mathrm{s}}\left(9+2 n_{\mathrm{s}}\right)\right) \widetilde{k}^{4}\right)\right),
\end{aligned}
$$

where $R\left(x_{\mathrm{R}}\right)$ is the relative suppression factor given by Eq. (45) of Ref. [10]. In the scale-invariant case $\left(n_{\mathrm{s}}=1\right)$, this reproduces the leading term in the large-scale limit $k \ll k_{\max }$ of the formula in Ref. [10] up to the factor $1 / 4$ revised in our accompanying paper [39]. The above formula is derived assuming $n_{\mathrm{s}}>1 / 2$. There are no resonance contributions for $\Omega_{\mathrm{GW}, \mathrm{eMD}}$, so that the total spectrum can be approximated by the above formula, $\Omega_{\mathrm{GW}, \mathrm{eMD}} \simeq \Omega_{\mathrm{GW}, \mathrm{eMD}}^{(\mathrm{LS})}$ for $k \ll k_{\max }$. However, this contribution is subdominant compared to $\Omega_{\mathrm{GW}, \mathrm{RD}}$ as shown in Fig. 1 for $n_{\mathrm{s}}=1$.
[1] B. P. Abbott et al. (Virgo, LIGO Scientific), Phys. Rev. Lett. 116, 061102 (2016), arXiv:1602.03837 [gr-qc].

[2] B. P. Abbott et al. (Virgo, LIGO Scientific), Phys. Rev. Lett. 119, 141101 (2017), arXiv:1709.09660 [gr-qc].

[3] T. Akutsu et al. (KAGRA), Nat. Astron. 3, 35 (2019), arXiv:1811.08079 [gr-qc].

[4] K. N. Ananda, C. Clarkson, and D. Wands, Phys. Rev. D75, 123518 (2007), arXiv:gr-qc/0612013 [gr-qc].

[5] D. Baumann, P. J. Steinhardt, K. Takahashi, and K. Ichiki, Phys. Rev. D76, 084019 (2007), arXiv:hepth/0703290 [hep-th].

[6] R. Saito and J. Yokoyama, Phys. Rev. Lett. 102, 161101 (2009), [Erratum: Phys. Rev. Lett.107,069901(2011)], arXiv:0812.4339 [astro-ph].

[7] R. Saito and J. Yokoyama, Prog. Theor. Phys. 123, 867 (2010), [Erratum: Prog. Theor. Phys.126,351(2011)], arXiv:0912.5317 [astro-ph.CO].

[8] L. Alabidi, K. Kohri, M. Sasaki, and Y. Sendouda, JCAP 1209, 017 (2012), arXiv:1203.4663 [astro-ph.CO].

[9] J. R. Espinosa, D. Racco, and A. Riotto, JCAP 1809, 012 (2018), arXiv:1804.07732 [hep-ph].

[10] K. Kohri and T. Terada, Phys. Rev. D97, 123532 (2018),
arXiv:1804.08577 [gr-qc].

[11] R.-g. Cai, S. Pi, and M. Sasaki, (2018), arXiv:1810.11000 [astro-ph.CO].

[12] N. Bartolo, V. De Luca, G. Franciolini, M. Peloso, and A. Riotto, (2018), arXiv:1810.12218 [astro-ph.CO].

[13] N. Bartolo, V. De Luca, G. Franciolini, M. Peloso, D. Racco, and A. Riotto, (2018), arXiv:1810.12224 [astro-ph.CO].

[14] C. Unal, Phys. Rev. D99, 041301 (2019), arXiv:1811.09151 [astro-ph.CO].

[15] C. T. Byrnes, P. S. Cole, and S. P. Patil, (2018), arXiv:1811.11158 [astro-ph.CO].

[16] K. Inomata and T. Nakama, Phys. Rev. D99, 043511 (2019), arXiv:1812.00674 [astro-ph.CO].

[17] S. Clesse, J. García-Bellido, and S. Orani, (2018), arXiv:1812.11011 [astro-ph.CO].

[18] R.-G. Cai, S. Pi, S.-J. Wang, and X.-Y. Yang, (2019), arXiv:1901.10152 [astro-ph.CO].

[19] Y.-F. Cai, C. Chen, X. Tong, D.-G. Wang, and S.-F. Yan, (2019), arXiv:1902.08187 [astro-ph.CO].

[20] S. Wang, T. Terada, and K. Kohri, (2019), arXiv:1903.05924 [astro-ph.CO]. 
[21] I. Ben-Dayan, B. Keating, D. Leon, and I. Wolfson, (2019), arXiv:1903.11843 [astro-ph.CO].

[22] Y. Tada and S. Yokoyama, (2019), arXiv:1904.10298 [astro-ph.CO].

[23] L. Lentati et al., Mon. Not. Roy. Astron. Soc. 453, 2576 (2015), arXiv:1504.03692 [astro-ph.CO].

[24] R. M. Shannon et al., Science 349, 1522 (2015), arXiv:1509.07320 [astro-ph.CO].

[25] Z. Arzoumanian et al. (NANOGrav), Astrophys. J. 821 , 13 (2016), arXiv:1508.03024 [astro-ph.GA].

[26] C. J. Moore, R. H. Cole, and C. P. L. Berry, Class. Quant. Grav. 32, 015014 (2015), arXiv:1408.0740 [gr-qc].

[27] G. Janssen et al., Proceedings, Advancing Astrophysics with the Square Kilometre Array (AASKA14): Giardini Naxos, Italy, June 9-13, 2014, PoS AASKA14, 037 (2015), arXiv:1501.00127 [astro-ph.IM].

[28] B. P. Abbott et al. (Virgo, LIGO Scientific), Phys. Rev. Lett. 120, 091101 (2018), arXiv:1710.05837 [gr-qc].

[29] Virgo, http://www.virgo-gw.eu/.

[30] B. S. Sathyaprakash and B. F. Schutz, Living Rev. Rel. 12, 2 (2009), arXiv:0903.0338 [gr-qc].

[31] Einstein Telescope, http://www.et-gw.eu/.

[32] B. P. Abbott et al. (LIGO Scientific), Class. Quant. Grav. 34, 044001 (2017), arXiv:1607.08697 [astro-ph.IM].

[33] H. Audley et al. (LISA), (2017), arXiv:1702.00786 [astroph.IM].

[34] N. Seto, S. Kawamura, and T. Nakamura, Phys. Rev. Lett. 87, 221103 (2001), arXiv:astro-ph/0108011 [astro$\mathrm{ph}]$.

[35] K. Yagi and N. Seto, Phys. Rev. D83, 044011 (2011), [Erratum: Phys. Rev.D95,no.10,109901(2017)], arXiv:1101.3940 [astro-ph.CO].

[36] E. Phinney et al., Big Bang Observer mission concept study (2003).

[37] H. Assadullahi and D. Wands, Phys. Rev. D79, 083511 (2009), arXiv:0901.0989 [astro-ph.CO].

[38] L. Alabidi, K. Kohri, M. Sasaki, and Y. Sendouda, JCAP 1305, 033 (2013), arXiv:1303.4519 [astro-ph.CO].

[39] K. Inomata, K. Kohri, T. Nakama, and T. Terada, (2019), arXiv:1904.12878 [astro-ph.CO].

[40] T. Nakama, J. Silk, and M. Kamionkowski, Phys. Rev. D95, 043511 (2017), arXiv:1612.06264 [astro-ph.CO].

[41] J. Garcia-Bellido, M. Peloso, and C. Unal, JCAP 1709, 013 (2017), arXiv:1707.02441 [astro-ph.CO].

[42] F. Arroja, H. Assadullahi, K. Koyama, and D. Wands, Phys. Rev. D80, 123526 (2009), arXiv:0907.3618 [astroph.CO].

[43] J.-C. Hwang, D. Jeong, and H. Noh, Astrophys. J. 842, 46 (2017), arXiv:1704.03500 [astro-ph.CO].

[44] N. Aghanim et al. (Planck), (2018), arXiv:1807.06209 [astro-ph.CO].
[45] S. Mollerach, D. Harari, and S. Matarrese, Phys. Rev. D69, 063002 (2004), arXiv:astro-ph/0310711 [astro-ph].

[46] V. Mukhanov, Physical Foundations of Cosmology (Cambridge Univ. Press, Cambridge, 2005).

[47] K. Ando, K. Inomata, and M. Kawasaki, Phys. Rev. D97, 103528 (2018), arXiv:1802.06393 [astro-ph.CO].

[48] K. Jedamzik, M. Lemoine, and J. Martin, JCAP 1009, 034 (2010), arXiv:1002.3039 [astro-ph.CO].

[49] K. Jedamzik, M. Lemoine, and J. Martin, JCAP 1004 , 021 (2010), arXiv:1002.3278 [astro-ph.CO].

[50] E. Thrane and J. D. Romano, Phys. Rev. D88, 124032 (2013), arXiv:1310.5300 [astro-ph.IM].

[51] M. Anholm, S. Ballmer, J. D. E. Creighton, L. R. Price, and X. Siemens, Phys. Rev. D79, 084030 (2009), arXiv:0809.0701 [gr-qc].

[52] X. Siemens, J. Ellis, F. Jenet, and J. D. Romano, Class. Quant. Grav. 30, 224015 (2013), arXiv:1305.3196 [astroph.IM].

[53] S. J. Chamberlin, J. D. E. Creighton, X. Siemens, P. Demorest, J. Ellis, L. R. Price, and J. D. Romano, Phys. Rev. D91, 044048 (2015), arXiv:1410.8256 [astro-ph.IM].

[54] P. A. R. Ade et al. (Planck), Astron. Astrophys. 594, A13 (2016), arXiv:1502.01589 [astro-ph.CO].

[55] P. F. de Salas, M. Lattanzi, G. Mangano, G. Miele, S. Pastor, and O. Pisanti, Phys. Rev. D92, 123534 (2015), arXiv:1511.00672 [astro-ph.CO].

[56] S. Saga, D. Yamauchi, and K. Ichiki, Phys. Rev. D92, 063533 (2015), arXiv:1505.02774 [astro-ph.CO].

[57] L. Kofman, A. D. Linde, X. Liu, A. Maloney, L. McAllister, and E. Silverstein, JHEP 05, 030 (2004), arXiv:hepth/0403001 [hep-th].

[58] L. Kofman, (2003), arXiv:astro-ph/0303614 [astro-ph].

[59] G. Dvali, A. Gruzinov, and M. Zaldarriaga, Phys. Rev. D69, 083505 (2004), arXiv:astro-ph/0305548 [astro-ph].

[60] M. Zaldarriaga, Phys. Rev. D69, 043508 (2004), arXiv:astro-ph/0306006 [astro-ph].

[61] L. Ackerman, C. W. Bauer, M. L. Graesser, and M. B. Wise, Phys. Lett. B611, 53 (2005), arXiv:astroph/0412007 [astro-ph].

[62] D. I. Podolsky, G. N. Felder, L. Kofman, and M. Peloso, Phys. Rev. D73, 023501 (2006), arXiv:hep-ph/0507096 [hep-ph].

[63] K. Kohri, D. H. Lyth, and C. A. ValenzuelaToledo, JCAP 1002, 023 (2010), [Erratum: JCAP1009,E01(2011)], arXiv:0904.0793 [hep-ph].

[64] G. Bertone, D. Hooper, and J. Silk, Phys. Rept. 405, 279 (2005), arXiv:hep-ph/0404175 [hep-ph].

[65] K. Enqvist and A. Mazumdar, Phys. Rept. 380, 99 (2003), arXiv:hep-ph/0209244 [hep-ph].

[66] P. Binetruy, A. Bohe, C. Caprini, and J.-F. Dufaux, JCAP 1206, 027 (2012), arXiv:1201.0983 [gr-qc]. 